Vietnam Journal of Mechanics, VAST, Vol.41, No. 2 (2019), pp. 127-139

DOI: https://doi.org/10.15625/0866-7136/12977

\title{
AN ENHANCED NODAL GRADIENT FINITE ELEMENT FOR NON-LINEAR HEAT TRANSFER ANALYSIS
}

\author{
Minh Ngoc Nguyen ${ }^{1, *}$, Tich Thien Truong ${ }^{1}$, Tinh Quoc Bui ${ }^{2}$ \\ ${ }^{1}$ Ho Chi Minh City University of Technology, Viet Nam \\ ${ }^{2}$ Tokyo Institute of Technology, Tokyo, Japan \\ *E-mail: nguyenngocminh@hcmut.edu.vn
}

Received: 19 July 2018 / Published online: 28 February 2019

\begin{abstract}
The present work is devoted to the analysis of non-linear heat transfer problems using the recent development of consective-interpolation procedure. Approximation of temperature is enhanced by taking into account both the nodal values and their averaged nodal gradients, which results in an improved finite element model. The novel formulation possesses many desirable properties including higher accuracy and higher-order continuity, without any change of the total number of degrees of freedom. The non-linear heat transfer problems equation is linearized and iteratively solved by the Newton-Raphson scheme. To show the accuracy and efficiency of the proposed method, several numerical examples are hence considered and analyzed.
\end{abstract}

Keywords: consecutive-interpolation procedure; heat transfer; nonlinear; Newton-Raphson.

\section{INTRODUCTION}

Heat transfer is an important phenomenon in engineering as temperature varies in both space and time. Working under undesirable temperature may reduce the durability of industrial components. Therefore, studying on heat transfer problems has become one of major topics in both industrial and academic communities. Although closed-form solutions derived by analytical approaches are available, they are relatively limited to some specific problems with relatively simple geometry and/or boundary conditions. For engineering applications, which usually include geometries with complicated shape as well as sophisticated boundary conditions, numerical methods have arisen as more suitable alternatives.

A numerical approach is expected to produce reliable results with reasonable computational cost, while implementation should also be convenient. The finite element method (FEM) has been shown to be one of the most popular numerical methods that has been constantly used for engineering problems. However, the FEM itself owns several inherent shortcomings [1], for example, the gradient fields (i.e. heat flux in the case of heat transfer problems) reproduced by FEM are non-physically discontinuous at node. Such a flaw requires treatment during post-processing. Issues of FEM have motivated

(C) 2019 Vietnam Academy of Science and Technology 
researchers to propose alternative numerical methods, for instances the boundary element method (BEM) [2,3], and meshfree methods [4-6]. Nevertheless, each method has its own advantages and disadvantages. The BEM is relatively fast as it does not need discretization inside the problem domain, however fundamental solutions for each specific problem are often essential. Finding such fundamental solutions is not a trivial task, especially in complicated applications. The class of meshfree methods possesses the advantage that the problem domains are discretized into scattered nodes while nodal connectivities, i.e elements, are no longer required. Therefore, it offers more flexibility during updating geometry or discretization, for example in case of mesh refinement or optimization problems. However, most of the meshfree methods do not possess the Kronecker-delta property, causing difficulties in enforcement of boundary conditions.

The recent development of consecutive-interpolation procedure (CIP) $[7,8]$ does not attempt to introduce an alternative method, rather it is an improved version of FEM. In this concept, all the advantages of FEM are preserved, such as simplicity and Kroneckerdelta property. By taking both the nodal values and the averaged nodal gradients into interpolation scheme, a finite element enhanced by CIP is able to reproduce smooth gradient fields, and increase accuracy. Furthermore, the total number of degrees of freedom are the same as in FEM, given the same mesh. Extension of CIP into three-dimensional finite elements was investigated by the authors $[9,10]$ for analysis of linear heat transfer and linear elasticity, in which advantages of the proposed approach over traditional FEM are verified. The thermal process cannot always be considered as linear. Nonlinearities are usually involved, for example, in cases of temperature-dependent thermal properties $[5,11,12]$ or during heat radiation $[13,14]$. In this paper, the ability of CIP-enhanced finite element method in dealing with non-linear analysis of heat transfer is studied.

The paper is organized as follows. After the Introduction, a brief review on CIP formulation is presented. In Section 3, numerical aspects of the proposed approach for non-linear heat transfer are discussed. Two examples of heat transfer with nonlinearities involved are considered in Section 4, demonstrating the efficiency of CIP-enhanced elements in this particular problem type. The last Section is reserved for Conclusions and Remarks.

\section{BRIEF ON CIP FORMULATION}

Details on the CIP formulation have been reported in our previous works, e.g., see Refs. [8-10]. In this Section, we briefly present fundamentals of the CIP. Let us consider a solid body occupying in the domain $\Omega$ bounded by $\Gamma$. The domain is discretized into non-overlapping sub-domains $\Omega_{e}$ namely finite elements. An arbitrary $u(\mathbf{x})$ defined in $\Omega$ can be approximated using CIP as

$$
u(\mathbf{x})=\sum_{I=1}^{n} R_{I}(\mathbf{x}) \hat{u}_{I}=\mathbf{R} \hat{\mathbf{u}},
$$

where $n$ is the number of nodes, $\hat{u}_{I}$ is the nodal value of function $u(\mathbf{x})$ at node $I$ (global index), and $R_{I}$ is the CIP shape function associated with node $I$. The vector of shape 
functions $\mathbf{R}$ is computed by [9]

$$
\mathbf{R}(\mathbf{x})=\sum_{I=1}^{n}\left(\phi_{I}(\mathbf{x}) \mathbf{N}^{[I]}+\phi_{I x}(\mathbf{x}) \overline{\mathbf{N}}_{. x}^{[I]}+\phi_{I y}(\mathbf{x}) \overline{\mathbf{N}}_{. y}^{[I]}+\phi_{I z}(\mathbf{x}) \overline{\mathbf{N}}_{. z}^{[I]}\right),
$$

in which $\mathbf{N}^{[I]}$ is the vector of Lagrangian shape functions evaluated at node $I ; \overline{\mathbf{N}}_{. x}^{[I]}, \overline{\mathbf{N}}_{. y}^{[I]}$, $\overline{\mathbf{N}}_{. z}^{[I]}$ are respectively the averaged derivative of Lagrangian shape functions with respect to $x-, y-$, and $z$-directions. The calculation is as follows

$$
\overline{\mathbf{N}}_{. x}^{[I]}=\sum_{e \in S_{I}}\left(w_{e} \cdot \mathbf{N}_{, x}^{[I][e]}\right)
$$

where $\mathbf{N}_{, x}^{[I][e]}$ is the derivative of $\mathbf{N}^{[I]}$ computed in element $e$, and $w_{e}$ is a weight function defined by

$$
w_{e}=\frac{\Delta_{e}}{\sum_{\bar{e} \in S_{I}} \Delta_{\bar{e}}}, e \in S_{I} .
$$

Here, $S_{I}$ is the set of elements interconnected at node $I . \Delta_{e}$ is a measure of the size of element $e$, which can be taken as the volume for a 3D element and area for a $2 \mathrm{D}$ element.

It is emphasized that the set of auxiliary functions $\phi_{I}, \phi_{I x}, \phi_{I y}, \phi_{I z}$ have to be seperately developed for each type of elements [7-9], which is an issue that limits the applicability of CIP. Recently, the bottleneck is resolved due to the introduction of a general formulation to determine auxiliary functions for a wide range of finite elements [9]. Given an element $e$ with ne number of nodes, the auxiliary functions associated with the local $i^{\text {th }}(i=1,2, \ldots, n e)$ is calculated by [9]

$$
\begin{gathered}
\phi_{i}(\mathbf{x})=N_{i}+N_{i}^{2}\left(\Sigma_{1}-N_{i}\right)-N_{i}\left(\Sigma_{2}-N_{i}^{2}\right), \\
\phi_{i x}(\mathbf{x})=\sum_{j=1, j \neq i}^{n e}\left(x_{j}-x_{i}\right)\left(N_{i}^{2} N_{j}+\frac{1}{2} N_{i} N_{j}\left(\Sigma_{1}-N_{i}-N_{j}\right)\right),
\end{gathered}
$$

where $N$ is the Lagrange shape functions. Quantities $\Sigma_{1}$ and $\Sigma_{2}$ are defined by

$$
\Sigma_{1}(\mathbf{x})=\sum_{i=1}^{n e} N_{i}, \quad \Sigma_{2}(\mathbf{x})=\sum_{i=1}^{n e} N_{i}^{2}
$$

Replacing the $x$-coordinates in Eq. (6) by $y$ - and $z$-coordinates, the functions $\phi_{i y}$ and $\phi_{i z}$ are obtained. With the above general formulation, it is able to incorporate CIP into a wide range of finite elements from one-dimensional to three-dimensional elements to develop a new class of CIP-enhanced elements. Indeed, CIP can be implemented as an add-on into existing FEM codes.

\section{HEAT TRANSFER PROBLEMS}

Being derived from energy conservation, the governing equation of heat transfer problem is written by

$$
\nabla \cdot(\mathbf{k} \nabla T)+Q=\rho c \frac{\partial T}{\partial t} .
$$


Without consideration of heat radiation, the following boundary conditions are given

$$
\begin{gathered}
T=\bar{T} \text {, on } \Gamma_{1} \text { : Dirichlet boundary, } \\
(\mathbf{k} \nabla T) \cdot \mathbf{n}=\bar{q} \text {, on } \Gamma_{2}: \text { Neumann boundary, } \\
(\mathbf{k} \nabla T) \cdot \mathbf{n}=h\left(T_{a}-T\right), \text { on } \Gamma_{3}: \text { convection boundary. }
\end{gathered}
$$

In Eq. (8), $\mathbf{k}=\operatorname{diag}\left(k_{x x}, k_{y y}, k_{z z}\right)$ is the tensor of conductivities; $T$ is the temperature; $Q$ is the heat sink/source; $t$ is time; $\rho$ and $c$ are the density and specific heat capacitance, respectively. In the boundary conditions in Eqs. (9)-(12), $\bar{T}$ is the prescribed temperature; $\bar{q}$ is the prescribed heat flux; $\mathbf{n}$ is the outward normal unit vector of the boundary; $T_{a}$ is the ambient temperature; $h$ is the coefficient of convection. Nonlinearity is implied in Eq. (12) due to the dependency of material parameters on temperature, for example conductivity.

By some mathematical manipulation, the partial differential Eq. (8) is transformed into weak formulation as follows

$$
\int_{\Omega} \rho c \frac{\partial T}{\partial t} \delta T d \Omega+\int_{\Omega}(\delta \nabla T) \mathbf{k} \nabla T d \Omega-\int_{\Omega} Q \delta T d \Omega-\int_{\Gamma} \bar{q} \delta T d \Gamma-\int_{\Gamma} h\left(T_{a}-T\right) \delta T d \Gamma=0 .
$$

The partial derivative of temperature with respect to time can be approximated by Backward-Euler scheme, which is known to be less vulnerable to spurious oscillation $[6,11,15]$

$$
\frac{\partial T}{\partial t}(t+\Delta t)=\frac{1}{\Delta t}(T(t+\Delta t)-T(t))
$$

Similar to the spatial domain, the time domain can also be discretized into many time steps. By knowing the temperature at the beginning of the simulation, i.e. the initial condition, temperature at any given time steps within the time domain can be solved. Solution of the nonlinear equation (13) is then obtained using the iterative Newton-Raphson scheme (see [16,17]). Convergence is achieved when the residual evaluated in Eq. (12) is less than a pre-set tolerance, which is chosen to be $10^{-6}$ in this paper. A detailed explanation on Newton-Raphson procedure and flowchart of the scheme are presented in the Appendix.

\section{NUMERICAL EXAMPLES}

Two numerical examples inluding one two-dimensional (2D) problem and one threedimensional (3D) problem are analyzed. The discretization of 2D domain is conducted by using the four-node quadrilateral element, in which $Q 4$ is denoted as the FEM version and CQ4, on the other hand, is the CIP-enhanced element. For 3D domain, the eight-node hexahedral is employed, in which $\mathrm{HH} 8$ and $\mathrm{CHH} 8$ are respectively the traditional FEM and the CIP-based element. In heat transfer problems, each node is associated with one degree of freedom (DOF), i.e. nodal temperature. 


\subsection{Transient heat conduction in a hollow cylinder}

The first example considers heat conduction in an aluminum hollow cylinder as shown in Fig. 1. Due to symmetry of the problem configuration, only one-fourth is thus modeled. Initially (at $t=0$ ), the temperature is $T_{0}=400 \mathrm{Kin}$ the whole cylinder. Then inner wall is prescribed by $T_{1}=600 \mathrm{~K}$, while the outer wall is thermally insulated. It is expected that the cylinder will be gradually heated from the inner wall to the outer wall. The dependency of specific heat capacity [18] and thermal conductivity [19] on temperature from $400 \mathrm{~K}$ to $600 \mathrm{~K}$ is presented in Tab. 1. Values that are not found in Tab. 1 will be linearly interpolated. Mass density is assumed to be constant: $\rho=2700 \mathrm{~kg} / \mathrm{m}^{3}$. This example serves to verify the applicability of the proposed method, i.e. the CIP-enhanced finite element, on analysis of nonlinear heat transfer.
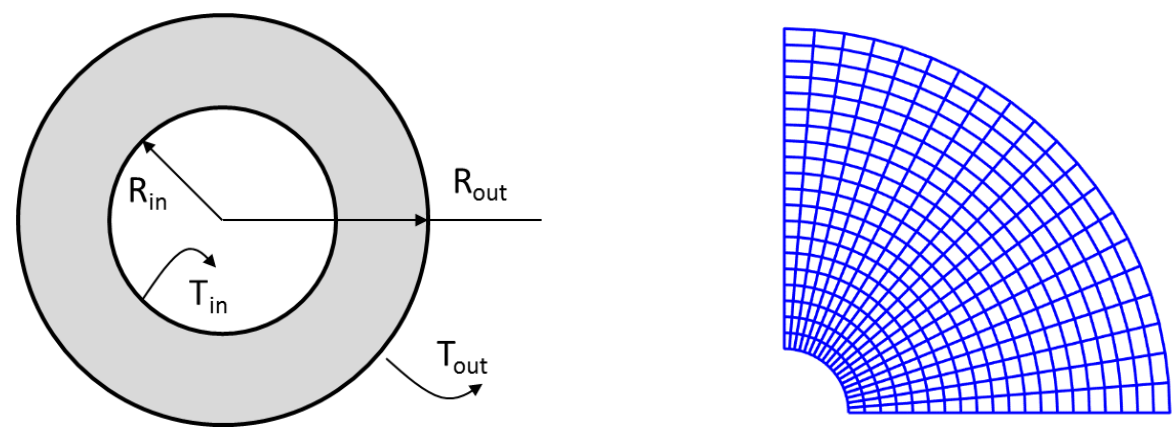

Fig. 1. Example 4.1. Geometry (left) and Finite element mesh for one-fourth of the cross-section (right)

Table 1. Example 4.1: Variation of temperature with respect to time

\begin{tabular}{ccc}
\hline Temperature $[\mathrm{K}]$ & Heat capacity $[\mathrm{J} /(\mathrm{kgK})]$ & Conductivity $[\mathrm{W} /(\mathrm{mK})]$ \\
\hline 400 & 951 & 240 \\
500 & 991.6 & 236 \\
600 & 1036.8 & 231 \\
\hline
\end{tabular}

For numerical analysis, a mesh of $20 \times 20$ quadrilateral element is used to discretize the spatial domain (one-fourth of the cross-section), see Fig. 1. The backward Euler time marching scheme is use for a total time span of 100 secs with 100 uniform time steps, i.e. time increment in each step is $\Delta t=1$ secs. Fig. 2 depicts the variation of temperature with respect to time. It is clearly observed that the temperature gradually increases from 400 $\mathrm{K}$ to $600 \mathrm{~K}$, starting from the inner wall to the outer wall of the cylinder. This observation is further supported by Fig. 3, which plots the variation of temperature along the line passing to the center and being inclined with horizontal direction an angle of $45^{\circ}$. In Fig. 3, temperature profiles are obtained by three levels of quadrilateral elements: $10 \times$ $10,20 \times 20$ and $40 \times 40$ elements. It is evidenced that results are mesh-independent. 

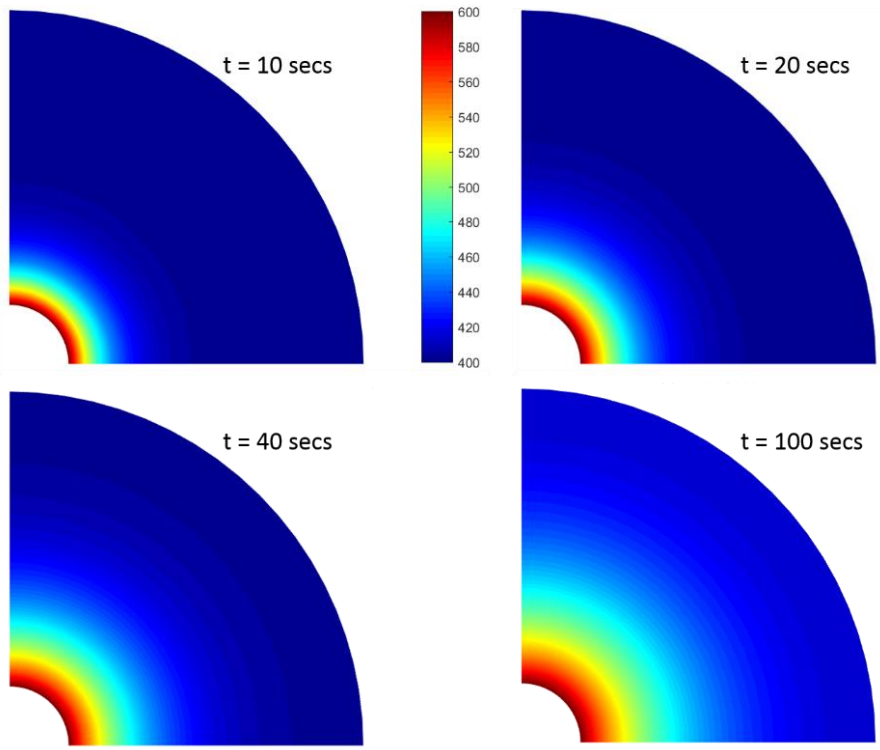

Fig. 2. Example 4.1: Variation of temperature with respect to time
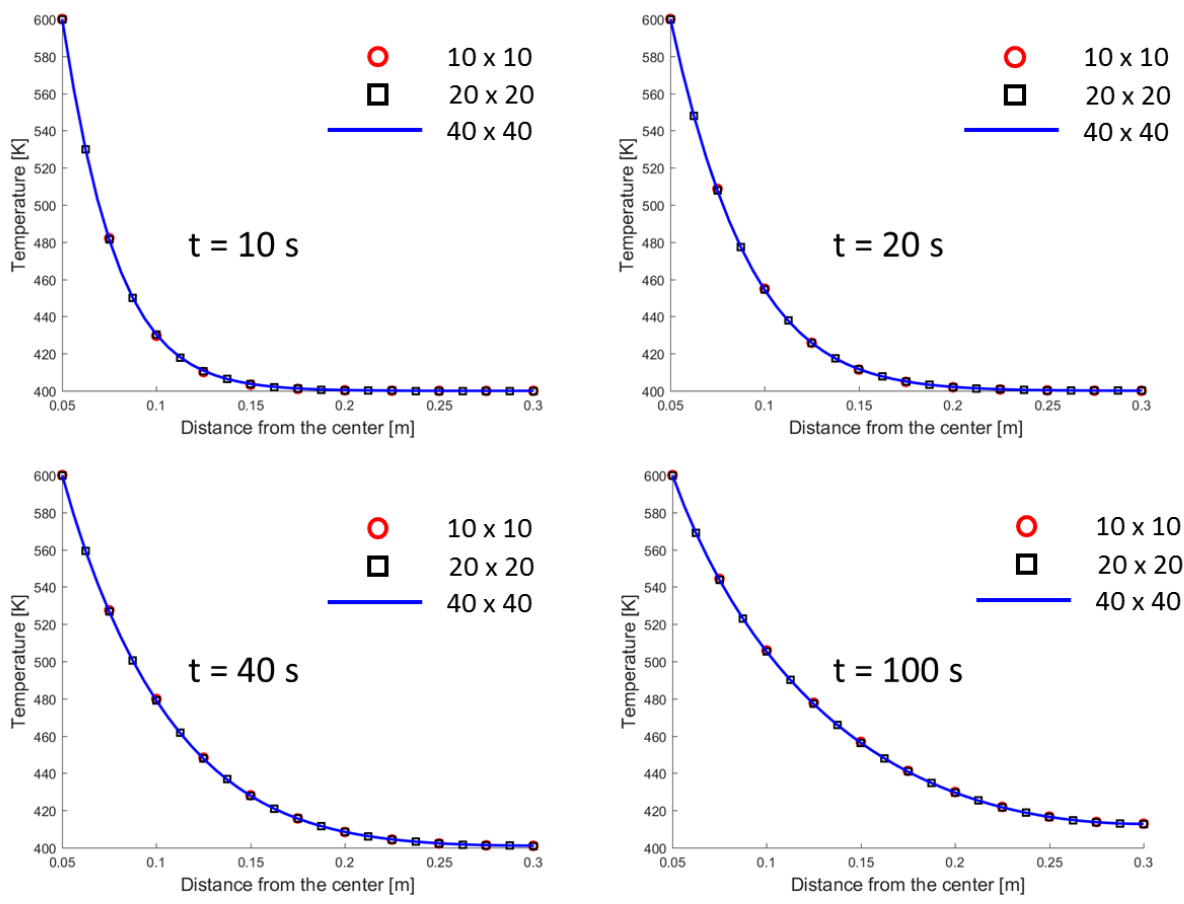

Fig. 3. Example 4.1: Variation of temperature about the line passing through center and being inclined with the horizontal direction by an angle of $45^{\circ}$ 
The desirable property of physically smooth gradient fields of CQ4, as reported in previous works for linear problems $[8,20]$ is still preserved, as depicted in Fig. 4 for $x$-component of heat flux. The field evaluated by Q4 elements are non-physically discontinuous, while that by CQ4 elements is continuous.

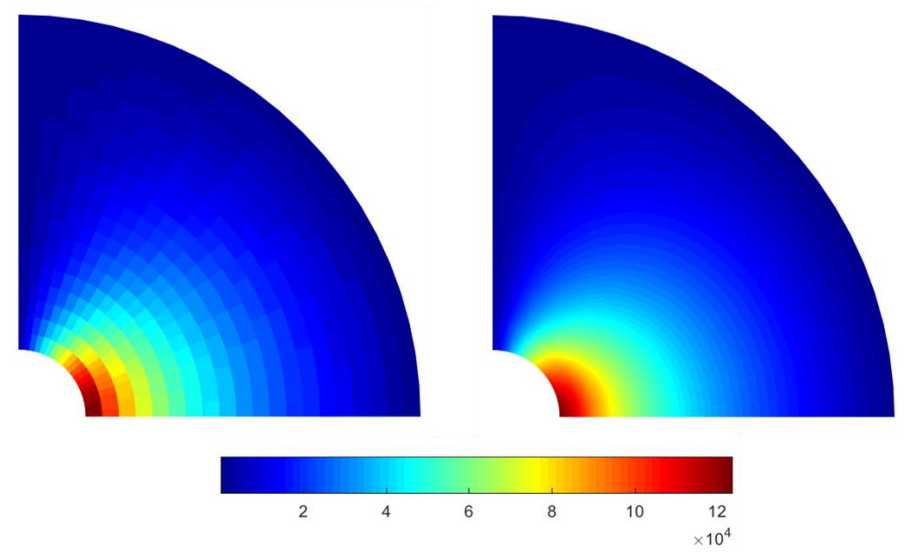

Fig. 4. Example 4.1: The $x$-component of heat flux provided by (left) Q4 elements and (right) CQ4 elements using the same mesh of $20 \times 20$ elements

\subsection{Transient heat transfer in a square plate with a cylindrical hole}

The second numerical example deals with transient heat transfer problem in a square plate with a cylindrical hole at center. The plate is subjected to both Dirichlet and Robin (i.e. convection) boundary conditions, as illustrated in Fig. 5. Similar to the previous example, only a quarter of the plate is modeled in this simulation due to the symmetry. Material properties on temperature are given as follows: thermal conductivity $k=15+$ $0.01 \mathrm{~T} \mathrm{~W} / \mathrm{m}^{\circ} \mathrm{C}$, mass density $\rho=7800-0.03 T \mathrm{~kg} / \mathrm{m}^{3}$, and specific heat capacitance $c=$ $125-0.015 T \mathrm{~J} / \mathrm{kg}^{\circ} \mathrm{C}$. Initially, the temperature of the entire domain is $T_{0}=50{ }^{\circ} \mathrm{C}$. The prescribed temperature on Dirichlet boundary, i.e. the wall of cylindrical hole, is $=200^{\circ} \mathrm{C}$.

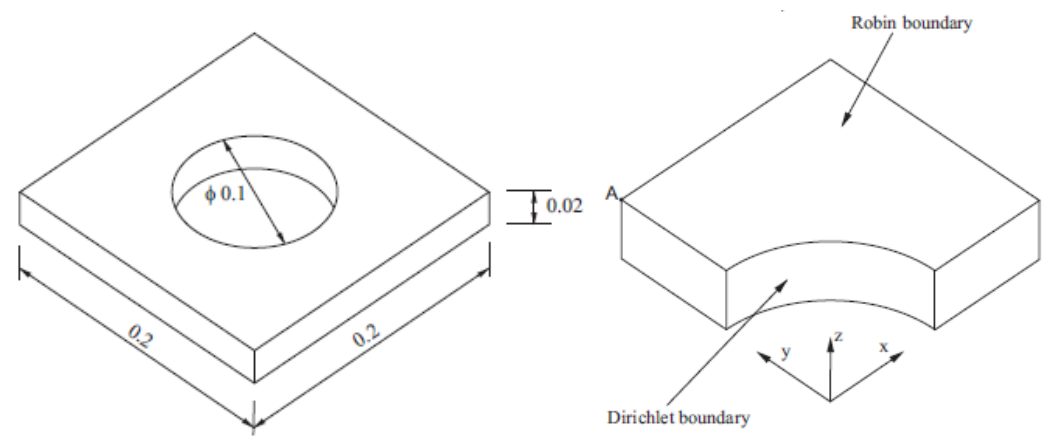

Fig. 5. Example 4.2: Full geometry (left) and one-fourth model due to symmetry (right) 
For the Robin condition, ambient temperature is $T_{a}=100{ }^{\circ} \mathrm{C}$ and convective coefficient is $h=200 \mathrm{~W} / \mathrm{m}^{2}$.

Three-level of hexahedral meshes are considered: 150 elements (264 nodes), 600 elements (924 nodes) and 2400 elements (3444 nodes). Fig. 6 visualizes the discretization of 600 elements. The time domain is divided into 100 time steps, with step size $\Delta t=7.5$ secs. After 750 secs, the solution can be considered as steady-state, which is clearly observed from Fig. 7, where the graph of evolution of temperature at point A (see Fig. 5 for position of A) is provided. The data obtained by the 600 elements and 2400 elements are almost identical, while the those by coarsest mesh are a little bit lower. These results evidently exhibit the mesh-independency of the temperature predicted by the proposed scheme. The temperature field at 750s computed by the $\mathrm{CHH} 8$ elements is depicted in Fig. 8.

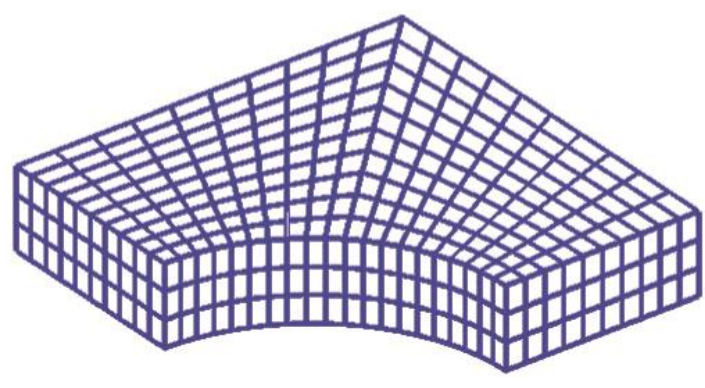

Fig. 6. Example 4.2: The mesh of 600 hexahedral elements

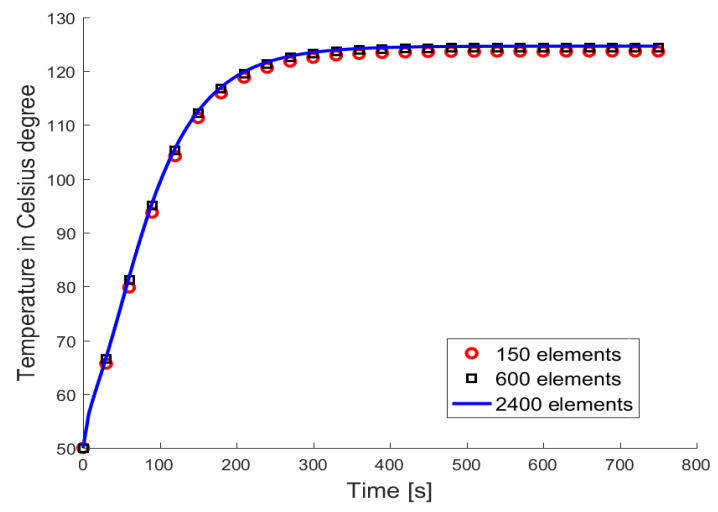

Fig. 7. Example 4.2: Evolution of temperature at point $\mathrm{A}$ (see Fig. 5)

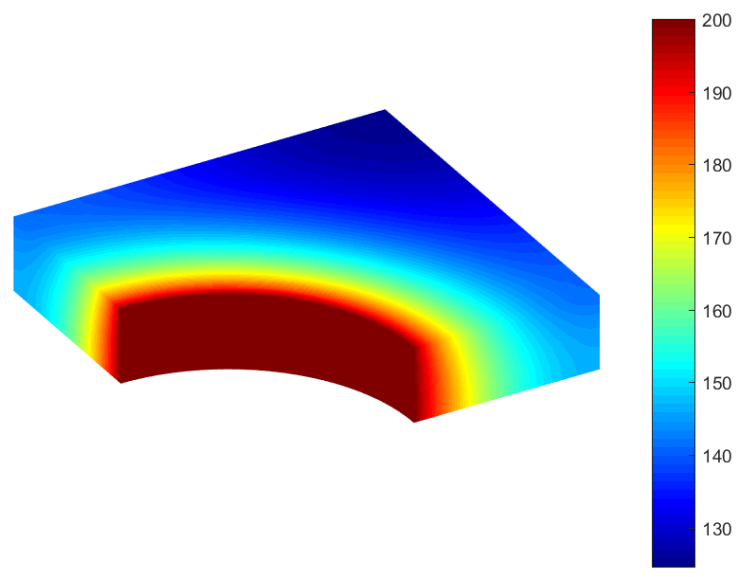

Fig. 8. Example 4.2: Steady-state temperature distribution (after 750 secs) 
Once temperature distribution is known, the equivalent thermal energy over the whole domain can be computed by

$$
U_{e}=\int_{\Omega}(\nabla T)^{T} k(\nabla T) d \Omega .
$$

In order to assess the performance of $\mathrm{CHH} 8$ element and the FEM counterpart, i.e. HH8 element, convergence of steady-state thermal energy (evaluated at $t=750 \mathrm{secs}$ ) with respect to mesh density is studied. To serve for comparison, the FEM solution obtained by the fine mesh of $38400 \mathrm{HH} 8$ elements (52164 nodes), i.e equivalent thermal energy is approximately $3533 \mathrm{~J} / \mathrm{m}^{3}{ }^{\circ} \mathrm{C}$, is adopted as reference value. The graph of numerical error versus number of DOFs (which is equal to the number of nodes) is depicted in Fig. 9, exhibiting that $\mathrm{CHH} 8$ has higher rate of convergence than $\mathrm{HH} 8$.

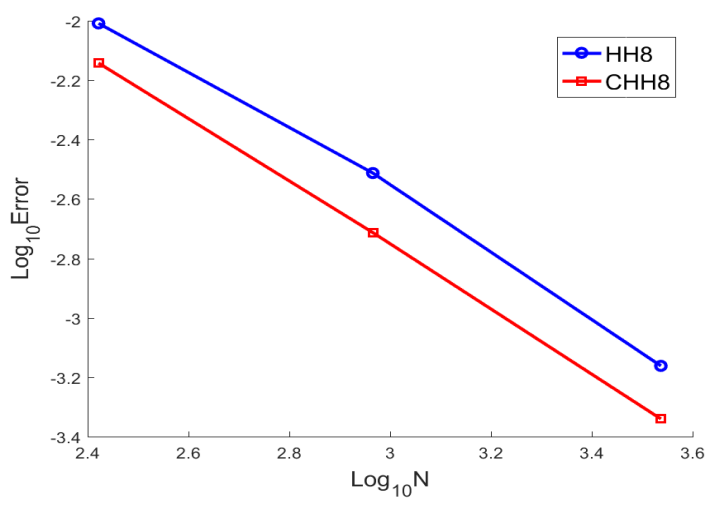

Fig. 9. Example 4.2: Accuracy of steady-state thermal energy with respect to mesh density, presented in log-log scale. Here $N$ is the number of DOFs (which is equal to the number of nodes)

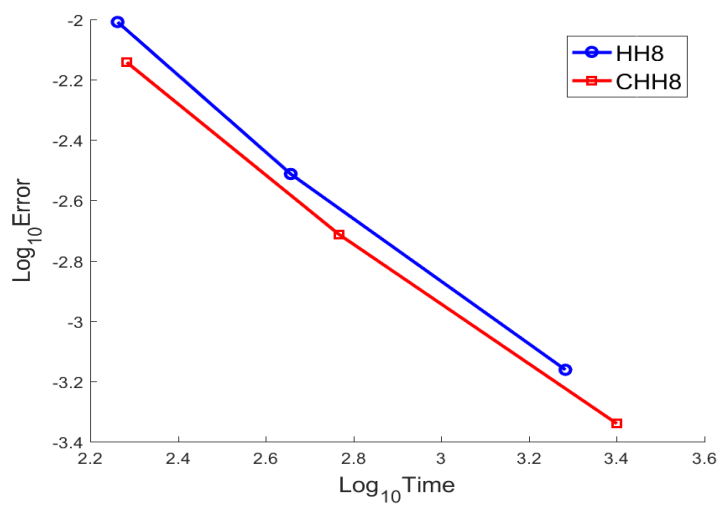

Fig. 10. Example 4.2: Comparison of accuracy achieved in term of computational time between HH8 and CHH8 elements, presented in $\log -\log$ scale

A comparison of accuracy achieved in term of computational time between $\mathrm{HH} 8$ and $\mathrm{CHH} 8$ elements is shown in Fig. 10, where the graphs are plotted in log-log scale. It is clearly demonstrated that the $\mathrm{CHH} 8$ is more time efficiency than the HH8.

\section{CONCLUSION AND OUTLOOKS}

In this research, the CIP-enhanced formulation for finite element analysis, in short CFEM, has been successfully extended for nonlinear transient heat transfer problems. Performance of CFEM on nonlinear analysis is so far still not reported in literatures. The proposed extension is achieved straightforwardly with the aid of backward Euler time marching scheme and Newton-Rapshon iteration to linearize and solve the governing equation. At each time step, only three to four iterations are needed to reach the chosen tolerance, i.e. $10^{-6}$ in this paper. This is as expected, due to the quadratic convergence of the Newton-Raphson scheme. 
The gradient fields obtained by traditional finite elements are non-physically discontinuous at node. The CIP-enhanced formulation, on the other hand, which is able to reproduce smooth gradient fields, and hence it offers better solutions.

One drawback of CIP-based approach is due to the fact that more computation is required to construct basis functions in comparison with that of the conventional FEM. Another drawback is the larger band of matrices due to the higher continuity. However, it should be noted that CIP-based elements are more computational efficiency, in terms of less DOFs needed (i.e. coarser mesh) to achieve the same accuracy, as already discussed in our previous works $[9,10,21]$. Additionally, in traditional FEM, further techniques are demanded to treat the non-physically nodal-discontinuous gradient fields during postprocessing, which are not necessary in CIP-based elements.

Though the current work focuses only in nonlinear transient heat transfer analysis, it would be able to apply the proposed formulation to other types of nonlinear problems without much difficulties. The positive results of the current research are preliminary for further investigation in heat transfer. A possible extension in future works would be the consideration of moving heat sources, which is essential in many engineering applications $[22,23]$. When a pulsed electrical source or laser is used to induce thermal shock, the non-Fourier heat equation [24,25], which include additional term containing the relaxation time, should be considered.

\section{ACKNOWLEDGMENT}

The authors are grateful to all the valuable comments given by anonymous reviewers, which greatly help to improve the manuscript.

This research is funded by Ho Chi Minh City University of Technology - VNU-HCM, under grant number T-KHUD-2018-21.

\section{REFERENCES}

[1] H. C. Huang and A. S. Usmani. Finite element analysis for heat transfer: theory and software. Springer-Verlag, London, (1994).

[2] L. C. Wrobel and C. A. Brebbia. Boundary element methods in heat transfer. Springer, (1992).

[3] I. Simoes, N. Simoes, A. Tadeu, M. Reis, C. A. B. Vasconcellos, and W. J. Mansur. Experimental validation of a frequency domain BEM model to study 2D and 3D heat transfer by conduction. Engineering Analysis with Boundary Elements, 36, (11), (2012), pp. 1686-1698. https://doi.org/10.1016/j.enganabound.2012.05.007.

[4] I. V. Singh. A numerical solution of composite heat transfer problems using meshless method. International Journal of Heat and Mass Transfer, 47, (10-11), (2004), pp. 2123-2138. https://doi.org/10.1016/j.ijheatmasstransfer.2003.12.013.

[5] A. Khosravifard and M. R. Hematiyan. A new method for meshless integration in 2D and 3D Galerkin meshfree methods. Engineering Analysis with Boundary Elements, 34, (1), (2010), pp. 30-40. https://doi.org/10.1016/j.enganabound.2009.07.008.

[6] X. Y. Cui, S. Z. Feng, and G. Y. Li. A cell-based smoothed radial point interpolation method (CS-RPIM) for heat transfer analysis. Engineering Analysis with Boundary Elements, 40, (2014), pp. 147-153. https://doi.org/10.1016/j.enganabound.2013.12.004.

[7] C. Zheng, S. C. Wu, X. H. Tang, and J. H. Zhang. A novel twice-interpolation finite element method for solid mechanics problems. Acta Mechanica Sinica, 26, (2), (2010), pp. 265-278. 
[8] T. Q. Bui, D. Q. Vo, C. Zhang, and D. D. Nguyen. A consecutive-interpolation quadrilateral element (CQ4): formulation and applications. Finite Elements in Analysis and Design, 84, (2014), pp. 14-31. https://doi.org/10.1016/j.finel.2014.02.004.

[9] M. N. Nguyen, T. Q. Bui, T. T. Truong, N. A. Trinh, I. V. Singh, T. Yu, and D. H. Doan. Enhanced nodal gradient 3D consecutive-interpolation tetrahedral element (CTH4) for heat transfer analysis. International Journal of Heat and Mass Transfer, 103, (2016), pp. 14-27. https://doi.org/10.1016/j.ijheatmasstransfer.2016.07.038.

[10] M. N. Nguyen, T. Q. Bui, T. T. Truong, S. Tanaka, and S. Hirose. Numerical analysis of 3-D solids and composite structures by an enhanced 8-node hexahedral element. Finite Elements in Analysis and Design, 131, (2017), pp. 1-16. https://doi.org/10.1016/j.finel.2017.04.002.

[11] S. Z. Feng, X. Y. Cui, A. M. Li, and G. Z. Xie. A face-based smoothed point interpolation method (FS-PIM) for analysis of nonlinear heat conduction in multimaterial bodies. International Journal of Thermal Sciences, 100, (2016), pp. 430-437. https://doi.org/10.1016/j.ijthermalsci.2015.10.017.

[12] K. Yang, W.-Z. Feng, J. Wang, and X.-w. Gao. RIBEM for 2D and 3D nonlinear heat conduction with temperature dependent conductivity. Engineering Analysis with Boundary Elements, 87, (2018), pp. 1-8. https://doi.org/10.1016/j.enganabound.2017.11.001.

[13] L. H. Liu and J. Y. Tan. Meshless local Petrov-Galerkin approach for coupled radiative and conductive heat transfer. International Journal of Thermal Sciences, 46, (7), (2007), pp. 672-681. https://doi.org/10.1016/j.ijthermalsci.2006.09.005.

[14] Y. Zhang, H.-L. Yi, and H.-P. Tan. Natural element method analysis for coupled radiative and conductive heat transfer in semitransparent medium with irregular geometries. International Journal of Thermal Sciences, 76, (2014), pp. 30-42. https://doi.org/10.1016/j.ijthermalsci.2013.08.013.

[15] M. N. Nguyen, T. Q. Bui, T. Yu, and S. Hirose. Isogeometric analysis for unsaturated flow problems. Computers and Geotechnics, 62, (2014), pp. 257-267. https://doi.org/10.1016/j.compgeo.2014.08.003.

[16] N. T. Phong, N. T. Trung, and N. P. Vinh. About an approximate method to solve the static boundary value problems in the isotropic hardening elastic-plastic solid. Vietnam Journal of Mechanics, 28, (2), (2006), pp. 74-82. https://doi.org/10.15625/0866-7136/28/2/5522.

[17] K. D. Nguyen, M. N. Nguyen, H. V. Cong, and H. Nguyen-Xuan. Isogeometric analysis of linear isotropic and kinematic hardening elastoplasticity. Vietnam Journal of Mechanics, 39, (1), (2017), pp. 51-68. https://doi.org/10.15625/0866-7136/7817.

[18] E. H. Buyco and F. E. Davis. Specific heat of aluminum from zero to its melting temperature and beyond. Equation for representation of the specific heat of solids. Journal of Chemical and Engineering Data, 15, (4), (1970), pp. 518-523. https://doi.org/10.1021/je60047a035.

[19] C. Y. Ho, R. W. Powell, and P. E. Liley. Thermal conductivity of the elements: a comprehensive review. Journal of Physical and Chemical Reference Data, 3, (1), (1974), pp. 1-810.

[20] T. Q. Bui, X. Zhang, S. Hirose, and R. C. Batra. Analysis of 2-dimensional transient problems for linear elastic and piezoelectric structures using the consecutive-interpolation quadrilateral element (CQ4). European Journal of Mechanics-A/Solids, 58, (2016), pp. 112-130. https://doi.org/10.1016/j.euromechsol.2016.01.010.

[21] M. N. Nguyen, T. Q. Bui, N. T. Nguyen, T. T. Truong, and L. Van Lich. Simulation of dynamic and static thermoelastic fracture problems by extended nodal gradient finite elements. International Journal of Mechanical Sciences, 134, (2017), pp. 370-386. https://doi.org/10.1016/j.ijmecsci.2017.10.022. 
[22] M. Shibahara and S. N. Atluri. The meshless local Petrov-Galerkin method for the analysis of heat conduction due to a moving heat source, in welding. International Journal of Thermal Sciences, 50, (6), (2011), pp. 984-992. https://doi.org/10.1016/j.ijthermalsci.2010.12.012.

[23] Y. Sun and X. Zhang. Heat transfer analysis of a circular pipe heated internally with a cyclic moving heat source. International Journal of Thermal Sciences, 90, (2015), pp. 279-289. https://doi.org/10.1016/j.ijthermalsci.2014.12.009.

[24] B.-L. Wang and J.-C. Han. A finite element method for non-Fourier heat conduction in strong thermal shock environments. Frontiers of Materials Science in China, 4, (3), (2010), pp. 226-233. https://doi.org/10.1007/s11706-010-0090-4.

[25] W. T. Zhao, J. H. Wu, and Z. Chen. Analysis of non-Fourier heat conduction in a solid sphere under arbitrary surface temperature change. Archive of Applied Mechanics, 84, (4), (2014), pp. 505-518. https://doi.org/10.1007/s00419-013-0814-x.

\section{APPENDIX}

Using the formulation in Eq. (1), the temperature and temperature gradient are approximated by

$$
\begin{aligned}
T(\mathbf{x}) & =\sum_{I=1}^{n} R_{I}(\mathbf{x}) \hat{T}_{I}=\mathbf{R} \hat{\mathbf{T}}, \\
\nabla T(\mathbf{x}) & =\left[\begin{array}{lll}
\frac{\partial T}{\partial x} & \frac{\partial T}{\partial y} & \frac{\partial T}{\partial z}
\end{array}\right]^{T}=\mathbf{B} \hat{\mathbf{T}} .
\end{aligned}
$$

Analogously, the test function and gradient of test function can be written as

$$
\delta \mathrm{T}(\mathbf{x})=\mathbf{R} \delta \hat{\mathbf{T}}, \quad \delta \nabla \mathrm{T}(\mathbf{x})=\mathbf{B} \delta \hat{\mathbf{T}} .
$$

Substitution of Eqs. (15)-(17) into Eq. (12) and approximate the time derivative of temperature using Eq. (13) yields the following equation at time step $n+1$

$$
\begin{aligned}
\mathbf{G}\left(T_{n+1}\right)= & \int_{\Omega} \mathbf{R}^{T} \rho c \frac{1}{\Delta t}\left(T_{n+1}-T_{n}\right) d \Omega+\int_{\Omega} \mathbf{B}^{T} \mathbf{k} \nabla T d \Omega \\
& -\int_{\Omega} \mathbf{N}^{T} Q d \Omega-\int_{\Gamma} \mathbf{N}^{T} \bar{q} d \Gamma-\int_{\Gamma} \mathbf{N}^{T} h\left(T_{a}-T_{n+1}\right) d \Gamma=0,
\end{aligned}
$$

where $T_{n+1}=T\left(t=t_{n+1}\right)$ is the temperature at time step $n+1$ and $T_{n}=T\left(t=t_{n}\right)$ is the temperature at previous time step, which is already known. Due to the dependency of material parameters on temperature, e.g. $\rho=\rho(T), c=c(T)$ and $\mathbf{k}=\mathbf{k}(T)$, Eq. (18) is non-linear. Applying the Newton-Raphson iterative scheme, the following linearized form with respect to the unknowns, i.e. the nodal values of temperature $\hat{\mathbf{T}}_{\mathrm{n}+1}$, is obtained at iteration step $k+1$

$$
\left(\mathbf{K}_{1}+\mathbf{K}_{2}+\mathbf{K}_{3}+\mathbf{K}_{4}+\mathbf{K}_{5}+\mathbf{K}_{6}\right) \cdot\left(\hat{\mathbf{T}}_{n+1}^{k+1}-\hat{\mathbf{T}}_{n+1}^{k}\right)=-\mathbf{G}\left(\hat{\mathbf{T}}_{n+1}^{k}\right),
$$

where

$$
\mathbf{K}_{1}=\int_{\Omega} \mathbf{R}^{T} \cdot \frac{1}{\Delta t} \cdot \frac{\partial \rho}{\partial T} \cdot c \cdot\left(T_{n+1}^{k}-T_{n}\right) \cdot \mathbf{R} d \Omega,
$$




$$
\begin{aligned}
& \mathbf{K}_{2}=\int_{\Omega} \mathbf{R}^{T} \cdot \frac{1}{\Delta t} \cdot \rho \cdot \frac{\partial c}{\partial T} \cdot\left(T_{n+1}^{k}-T_{n}\right) \cdot \mathbf{R} d \Omega, \\
& \mathbf{K}_{3}=\int_{\Omega} \mathbf{R}^{T} \cdot \frac{1}{\Delta t} \cdot \rho \cdot c \cdot \mathbf{R} d \Omega, \\
& \mathbf{K}_{4}=\int_{\Omega} \mathbf{B}^{T} \cdot \mathbf{k} \cdot \mathbf{B} d \Omega, \\
& \mathbf{K}_{5}=\int_{\Omega} \mathbf{B}^{T} \cdot \frac{\partial \mathbf{k}}{\partial T} \cdot \nabla T_{n+1}^{k} \cdot \mathbf{R} d \Omega, \\
& \mathbf{K}_{6}=\int_{\Gamma} \mathbf{R}^{T} \cdot h_{a} \cdot \mathbf{R} d \Gamma .
\end{aligned}
$$

Notice that the term $\mathbf{K}_{6}$ only exists when the convection boundary condition is taken into account.

The nodal values of temperature at time step $\mathrm{n}$ is chosen as the starting guess for the time step $n+1$, i.e. $\hat{\mathbf{T}}_{n+1}^{0}=\hat{\mathbf{T}}_{n}$. Then Eq. (19) allows the iteratively update of the solutions. The solution at time step $n+1$ is said to be converged if the residual of Eq. (18) is less than a pre-defined tolerance, which is chosen to be $10^{-6}$ in this paper.

At each time step, if converged solution cannot be achieved after 15 iterations, the time step is considered as "Not converged" and will be re-calculate with smaller time increment. If the time increment is smaller than a pre-set "minimum time increment" $\Delta t_{\min }$ then the procedure stops due to non-convergence.

In summary, a flowchart is presented in Fig. 11.

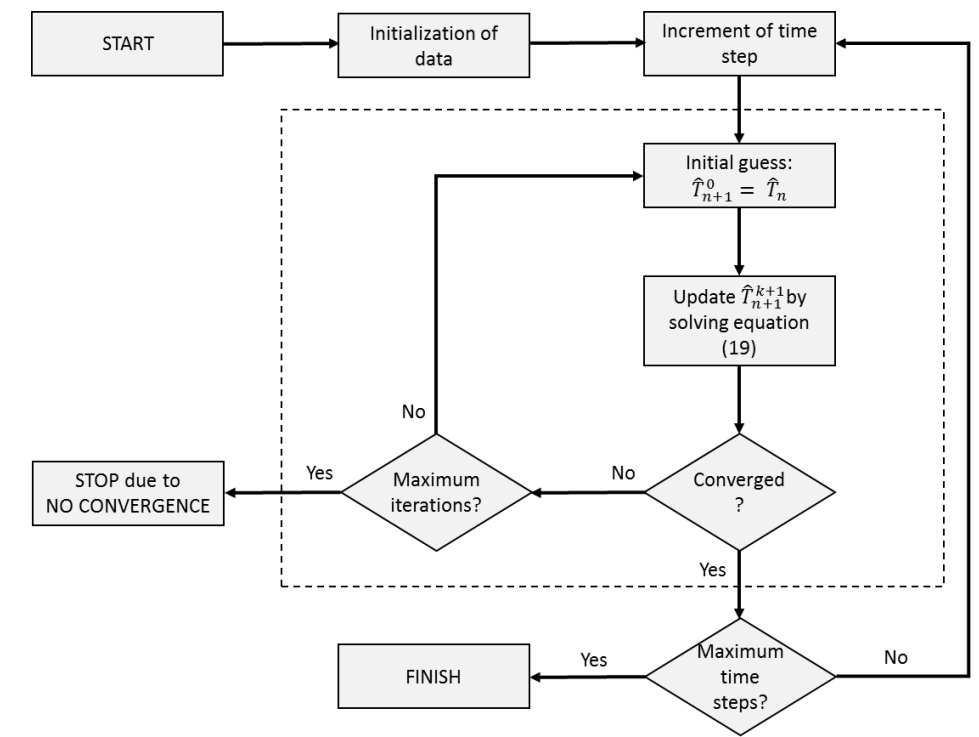

Fig. 11. Flowchart of numerical procedure for non-linear analysis of heat transfer 